\title{
Feasibility and outcome of substitution treatment of heroin-dependent patients in specialized substitution centers and primary care facilities in Germany: A naturalistic study in 2694 patients
}

\author{
Hans-Ulrich Wittchen ${ }^{\mathrm{a}, *}$, Sabine M. Apelt ${ }^{\mathrm{a}}$, Michael Soyka ${ }^{\mathrm{b}}$, Markus Gastpar ${ }^{\mathrm{d}}$, Markus \\ Backmund $^{\mathrm{f}}$, Jörg Gölz ${ }^{\mathrm{g}}$, Michael R. Kraus ${ }^{\text {h }}$, Felix Tretter ${ }^{\mathrm{i}}$, Martin Schäfer ${ }^{\mathrm{j}, \mathrm{k}}$, Jens Siegert ${ }^{\mathrm{a}}$, \\ Norbert Scherbaum ${ }^{1}$, Jürgen Rehm ${ }^{\text {a,e }}$, Gerhard Bühringer ${ }^{\text {a,c }}$
}

a Institute of Clinical Psychology and Psychotherapy, Technische Universitaet Dresden, Chemnitzerstrasse 46, 01187 Dresden, Germany

b Psychiatric Hospital, University of Munich, Nussbaumstrasse 7, 80336 Munich, Germany

c IFT Institute for Therapy Research, Parzivalstrasse 25, 80804 München, Germany

d Fliedner Klinik Berlin, Charlottenstrasse 65, 10117 Berlin, Germany

e Centre for Addiction and Mental Health, 33 Russell Street, Toronto, Ontario, Canada M5S 2S1

f Institut für Suchtmedizin und Adipositas, Tal 9, 80331 München, Germany

g Praxiszentrum Kaiserdamm, Kaiserdamm 24, 14057 Berlin, Germany

h Kreiskliniken Altötting-Burghausen, Krankenhausstrasse 1, 84489 Burghausen, Germany

i Isar-Amper-Klinikum, Klinikum München-Ost, Kompetenzzentrum Sucht, Postfach 1111, 85529

München Haar, Germany

j Klinik für Psychiatrie, Psychotherapie und Suchtmedizin, Kliniken-Essen-Mitte, Henricistrasse 92,

45136 Essen, Germany

k Klinik für Psychiatrie und Psychotherapie, Charit’e Campus Mitte, Charité Universitätsmedizin

Berlin, Charitéplatz 1, 10117 Berlin, Germany

1 Klinik für abhängiges Verhalten und Suchtmedizin, Rheinische Kliniken Essen, Institut der

Universität Duisburg-Essen, Virchowstrasse 174, 45147 Essen, Germany

\begin{abstract}
Background: In many countries, buprenorphine and methadone are licensed for the maintenance treatment (MT) of opioid dependence. Despite many short-term studies, little is known about the longterm (12-month) effects of these treatments in different settings, i.e. primary care-based (PMC) and specialized substitution centers (SSCs).

Objectives: To describe over a period of 12 months: (1) mortality, retention and abstinence rates; (2) changes in concomitant drug use, somatic and mental health; and (3) to explore differences between different types of provider settings.

Methods: 12-Month prospective-longitudinal naturalistic study with four waves of assessment in a prevalence sample of $\mathrm{N}=2694$ maintenance patients, recruited from a nationally representative sample of $\mathrm{N}=223$ substitution physicians.

Results: The 12 -month retention rate was $75 \%$; the mortality rate $1.1 \% .4 .1 \%$ of patients became "abstinent” during follow-up. 7\% were referred to drug-free addiction treatment. Concomitant drug use decreased and somatic health status improved. No significant improvements were observed for mental health and quality of life. When controlling for initial severity, small PMC settings revealed better retention, abstinence and concomitant drug use rates.

Conclusion: The study underlines the overall 12-month effectiveness of various forms of agonist MT. Findings reveal relatively high retention rates, low mortality rates, and improvements in most 12month outcome domains, except for mental health and quality of life. PMC settings appear to be a good additional option to improve access to MTs.
\end{abstract}

Keywords: Methadone; Buprenorphine; Epidemiology; Opioid dependence; Course; Outcome 


\section{Introduction}

Considerable changes have occurred in the treatment and care structure for opioid addicts over the past decade. In addition to a wide range of existing drug-free psychosocial abstinence programs (McLellan et al., 1993; Vollmer and Krauth, 2001), increased emphasis was placed in the last decade on establishing outpatient methadone maintenance therapy (MMT) and buprenorphine maintenance therapy (BMT) for the management of opioid-dependent individuals. Maintenance treatments (MTs) are provided by either large-scale specialized substitution centers (SSCs) or, more recently, by office-based physicians who either specialize fulltime on MMT/BMT treatment provision or who treat a few maintenance patients only, mostly within the context of their main function as primary care doctors (Gossop et al., 2003; Merrill, 2002; Wittchen et al., 2005). The primary short-term goals of treatment are retention in treatment, reduction of medical and social-behavioral risks, reduction of harm and mortality associated with injecting and other concomitant drug use, and interruption of the vicious circles of drug intake and criminal acts. By involving patients in a continuous treatment plan, it is also expected to reduce the burden of associated mental and somatic morbidities (psychosis, depression, HIV, viral hepatitis, etc.) and the substantial social sequelae as intermediate goals. The controversial long-term goal of maintenance regimen is in some countries explicitly - to help patients to ultimately quit the use of drugs entirely (abstinence) and to prepare them for the decision to enroll in a drug-free abstinence program. However, due to the lack of long-term data, it is unclear to what degree this goal is realistic.

Agonist maintenance therapies with methadone or more recently buprenorphine are currently the two most frequently used maintenance treatment strategies for opioid dependence in the care system. In clinical trials, both flexible-dose MMT and BMT have been shown to be consistently effective in at least reducing illicit opioid use (Johnson et al., 1992; Ling et al., 1976; Strain et al., 1994; Connock et al., 2007). In combination with various degrees of psychosocial support and psychological treatment other beneficial outcomes have also been demonstrated in clinical studies (review by Connock et al., 2007;Wittchen et al., 2005; Gerra et al., 2004; Gossop et al., 2001; Kakko et al., 2003; King et al., 2002; Layson-Wolf et al., 2002; Ling and Wesson, 2003; Mattick et al., 2003, 2004; Poser and Poser, 1996; Soyka et al., 1997; Waal and Haga, 2003), for example, with regard to (a) improvement of the social situation, (b) reduction of drug-related crime, (c) reduction of morbidity and mortality rates, and (d) reduction of the transmission rate of HIV.

Despite a considerable body of research (i.e. Connock et al., 2007; Gossop et al., 2001, 2003; King et al., 2002), several significant research deficits impede further progress. First, a lack of longitudinal studies in samples of unselected substitution settings and unselected samples of patients that inform us about how MT works under routine care conditions (Law and Nutt, 2003; Connock et al., 2007) and about the degree to which the assumed intermediate and long-term goals are reached in everyday practice. This deficit is regarded as an obstacle for the wider implementation of maintenance programs (Lawand Nutt, 2003).

Second, there is some evidence that the beneficial effects of MMT and BMT might be robust across different types of treatment settings. However the effects of different provider models and formats remain understudied, especially with regard to subgroups of addicts. Most available studies were undertaken in a few countries (US, UK, Australia) that have considerably different regulations and traditions than other countries (Connock et al., 2007). The German treatment system, for example, has been relatively slow in adopting MMT and BMT as first-line treatments for opioid-dependent individuals. Due to the existence of a wide 
range of specialized in- and outpatient long-term treatment programs established in the 1970s and 1980s, MMT programs were quite rare until the 1990s and were mainly provided by relatively few SSCs with quite restrictive regulations of access (Vollmer and Krauth, 2001; Wittchen et al., 2005). Although access to treatment programs remain up to now heavily regulated and rigidly controlled with quite restrictive criteria and quality assurance measures for access and management of patients, the number of institutions licensed to administer MMT and BMT have been largely increased during the past decade. Currently (2005) in Germany there are about 2500 medical doctors (with more than 65,000 maintenance patients), trained and licensed for MT, constituting a twofold increase over the rates before the year 2000 (Wittchen et al., 2005). This increase is mainly due to increasing number of smaller office-based physicians providing maintenance treatment. Stimulated by the availability of buprenorphine (Farell et al., 2000) and by evidence that maintenance in primary care settings may work (Gossop et al., 2001, 2003; Merrill, 2002; Salsitz et al., 2000), MMT and BMT in particular is increasingly offered by primary care doctors (PMC settings) or less frequently by psychiatrists and other specialists that frequently do not generally specialize on MMT and BMT, but rather manage only a few such patients within the context of their predominant and main function as a family doctor or specialist. Such PMC settings might differ in a number of aspects (e.g. climate, resources, expertise) that can be expected to affect quality of care and outcome. For example they typically do not have additional resources in terms of time, personnel and expertise to directly offer particularly the mandatory psychological and social interventions. Irrespective of this, these doctors have to follow, though, the same stringently enforced complex legislative regulations as specialized MT centers for example by collaboration with other institutions. The relative risks and benefits of maintenance treatments in such PMC contexts remain clearly understudied (Wittchen et al., 2005; Merrill, 2002).

Third, evidence is also lacking with regard to the relative benefits of methadone (preferred in SSC) and buprenorphine (preferred in PMC) (see Walsh et al., 1994; Groß and Soyka, 1999; Mintzer and Stitzer, 2002; Soyka et al., 2000; Connock et al., 2007; Wittchen et al., 2005).

This naturalistic study describes the course and outcome of patients in routine maintenance treatment in Germany based on a prospective-longitudinal 12-month observational epidemiological study design in a nationally representative sample of maintenance settings, and a random sample of their patients. This paper is the first publication from this project that will ultimately stretch over 5 years. The following questions are addressed: (1) What is the 1year outcome in terms of retention, mortality, and abstinence (respectively transfer to abstinence treatment)? (2) What is the 1-year outcome in terms of the reduction of concomitant drug use and improvement of patients' somatic and mental health status? (3) Are there indications that the type of setting in which the treatment is provided has an effect on the 1-year outcome of maintenance treatment?

\section{Methods}

\subsection{Design}

The study is an observational 12-month prospective-longitudinal study in a nationwide representative sample of all substitution doctors in Germany and an unselected prevalence sample of patients currently in maintenance treatment (for an overview of methods see Wittchen et al., 2005). It included a comprehensive baseline and a 12-month follow-up assessment of patients. Additionally, course and retention as well as drop out and reason for discontinuation were monitored in 3-month intervals. Assessments consisted of a self-report patient questionnaire, urine tests, and a comprehensive clinical interview and treatment 
documentation by the treating physician. The design also included a prestudy of all substitution doctors to assess characteristics of settings, with regard to training, treatment expertise, practice characteristics, and attitudes and preferences regarding treatment of opioid addicts (Kintzel, 2007).

\subsection{Study participants}

2.2.1. Baseline sampling and inclusion criteria. Based on a nationwide register of over 2500 licensed and registered substitution doctors meeting the rigid mandatory training requirements in Germany in 2003, a random sample of 379 doctors was invited of which 223 participated (response rate: $58.8 \%$ ). The sample was stratified to include a sufficiently high number of different settings and their patients. Settings ranged from office-based, mostly primary care (PMC), settings, managing only a few maintenance patients (small PMC: up to 10 patients a day, mean $n=5$ patients; $n=86$ ) as part of their main role as either family physicians $(82.8 \%)$ or psychiatrist/neurologist (14\%), through office-based physicians providing predominantly or fulltime MMT or BMT (medium PMC: $10-40$ patients a day, mean $n=26$ patients; $n=101$ ), to largescale, specialized substitution centers (SSCs: 40+ patients a day, mean $n=118$ patients; $n=36$ ). In Germany all doctors providing MT, are obliged by law (BtMG, Betäubungsmittelgesetz) to complete an addiction medicine curriculum, need a license to conduct treatment and prescribe opiates, and have to document for each patient, that all criteria for inclusion (e.g. manifest opiate dependence, repeated unsuccessful attempts to achieve abstinence, drug-free treatment not possible) are met. Further a comprehensive treatment plan has to be presented, including insurance of the mandatory psychosocial interventions. Throughout treatment a full documentation of treatment activities including mandatory regular urine tests for concomitant use (leading to cessation of MMT/BMT treatment), dosages of substitution drug and other prescriptions as well as treatment progress is required and enforced (quality assurance programs), with the ultimate explicit goals of abstinence or change to a drug-free long-term program.

As shown in Table 1, despite these mandatory rules there are some noteworthy differences between the three different types of settings considered. Large SSCs are characterized by a higher proportion of medical specialists, especially psychiatrists/neurologists ( $<<0.001)$, and have more staff (social workers, psychologists) enabling them to conduct psychosocial treatment components more intensively and more frequently within rather than outside the setting $(\mathrm{p}<0.001)$. By definition they have considerably larger number of patients. They conduct urine test under supervision, however, less frequently than the other settings, and allow only a small number of patients to have take-home prescriptions. Patients in all types of settings typically receive their medication in the doctor office; take-home prescriptions are typically limited to weekends. Settings do not differ with regard to doctors mean years of experience with substitution treatment or with regard to the average face-to-face duration of visits.

A total of $n=2694$ patients were enrolled from these 233 settings at baseline. The total baseline response rate of all eligible patients was $71.7 \%$ and was highest in the small PMC settings (82\% enrolled, $\mathrm{n}=849$ patients) and slightly lower (76\%) in medium PMC settings (enrolled, $n=1.269$ ) and the large SSC (72\% enrolled, $n=576$ ) settings (Wittchen et al., 2005). To reduce potential selection bias, recruitment was based on total listing by name initials and age of all maintenance patients on the pre-determined recruitment day. Small settings with less than 10 substitution patients per day were requested to approach all patients for enrolment. In settings with more than 10 listed patients per day, the study center randomly selected every second/respectively third or fourth, etc., attending patient on the assessment 
day. The enrolment and all assessment procedures were checked by external monitors, for example by comparing the initial total list patient characteristics with those on the subsequent patient assessment forms.

It should be noted that this was a prevalence sample and thus patients were recruited into the study regardless of the duration of their current treatment. Baseline assessment refers to the moment that the patients entered the study and not to the moment they entered the current treatment episode.

Inclusion criteria: All consecutive patients on the recruitment list of at least 16 years of age with current opioid dependence who were currently in agonist maintenance therapy with either buprenorphine or methadone were eligible for the study. Exclusion criteria included all patients with acute medical emergencies ( $\mathrm{n}$ excluded $=11$ ), patients with cognitive impairments severe enough to compromise meaningful completion of the self-report forms (n $=21$ ) and unwillingness to comply with study procedures including the mandatory urine tests $(n=17)$. Each patient gave written informed consent, as approved by the Ethics Committee of the Medical Faculty, Technische Universität Dresden, Germany.

\subsection{Follow-up waves, reasons for non-participation and drop out}

Fig. 1 describes the flow of the original $N=2694$ patients from baseline through the follow-up period and the respective intermediate assessments. A total of $\mathrm{N}=233$ patients were lost during the 12-month observation period. Overall $n=29$ doctors withdrew their study participation for various reasons (too much burden, stopped offering maintenance treatments, etc.) at some point during the observational period; for theses patients, no course and outcome information is available. Thus, information was documented for $\mathrm{N}=2461$ patients (conditional response rate: $91.4 \%$ ). For $\mathrm{N}=1631$ out of the 2461 patients the final comprehensive 12-month follow-up investigation was completed. Of these, $n=1248$ were treated with methadone, $n=367$ with buprenorphine and $n=19$ with codeine (not further dealt with in this paper). Thus, the remaining total baseline $\mathrm{N}$ was 2442, respectively $\mathrm{n}=$ 1615). For 827 patients information was only obtained from the intermediate assessments: 27 patients had died (one additional case treated with codeine), and 272 patients completed the maintenance treatment within the observation period, either because of having become "clean" (=successful termination of treatment) or because they were referred to an abstinence treatment setting. Additional interviews with physicians were conducted when necessary to ascertain patients “abstinence” status (see outcome criteria).

$\mathrm{N}=254$ patients discontinued the treatment within the observation period, because of changes of residence, or being imprisoned for longer periods of time or because of change of the maintenance treatment (change in doctor and type of substitution drug). In an additional $n=$ 119 patients the treating physician decided to discontinue the maintenance therapy because of disciplinary reasons, mostly because of serious concomitant drug use. A further $n=155$ discontinued the treatment on their own at some point in the 12-month period for various other reasons. Analyses reported in this paper are thus based on $n=2442$ patients, for whom baseline and course information was obtained. Separate analyses were also run for $n=1615$ patients that were still in treatment at the 12-month follow-up assessment (thus excluding those who were either not retained in treatment, died, or became abstinent) for which a complete baseline and follow-up documentation was available.

\subsection{Assessment}


After presentation of information about the study by the doctor and completion of the informed consent form, patients were asked to complete a 12-page patient questionnaire. The questionnaire consisted largely of various components of established instruments such as item groups of the EuropASI (European Addiction Severity Index) (Gsellhofer et al., 1999) and modules of the substance use questions of the WHO Composite International Diagnostic Interview (CIDI) (Wittchen et al., 1998). The patient questionnaire covered the following domains: (i) basic biosocial and socio-demographic information, (ii) social and legal life developmental history and status ratings, (iii) past and current drug use and illness history module (CIDI), (iv) mental health and substance use diagnostic status (DSM-IV substance use and other mental disorders by CIDI), (v) self-reported physical disorders (e.g. hepatitis C, HIV), (vi) past and current social role impairments, disabilities and problems specific to drug use, (vii) past and current treatment history, (viii) met and unmet subjective needs, (ix) current and past experiences with treatments and (x) quality of life (Greiner et al., 2003), and (xi) risk behaviors (e.g. needle sharing, unprotected sexual behavior). Feasibility and 1-week testretest reliability of the patient questionnaire was examined in a unselected sample of $n=60$ patients recruited from five of the settings under study conditions (Kintzel, 2007). The overall completion time averaged (mean) 52.4 min (range 32-86 min). Percentage agreement was generally high (78-100\%) with kappa values ranging for categorical variables from a low of .52-.60 for selected unmet needs questions and risk behaviors to a high of .90 and above for social role impairments. For continuous measures (e.g. age of onset, duration, frequency questions) intraclass coefficients (ICC) ranged from .70 to 1.0.

\subsubsection{Clinical interview and assessment. Each patient was evaluated by the doctor using a} standardized interviewand appraisal covering: (i) current and past maintenance treatments along with documentation of onset and interruption of all lifetime treatment episodes (e.g. dosage, dosing status), (ii) licit and illicit substance use behaviors and substance use, including severity ratings, (iii) past and current physical and mental disorders rated by severity using the clinical global impression scale (CGI) (Guy, 1976), and current and past treatments (e.g. mental and selected somatic disorders (e.g.HCVand HIV status), (iv) multidimensional evaluation of social and psychological functioning, (v) past and ongoing current maintenance-related interventions, (vi) compliance and problems of management, (vii) individual treatment targets, (viii) an abbreviated EuropASI rating to assess treatment needs and (ix) ratings of health risk behaviors (needle sharing, etc.). No formal training was provided for the EuropASI administration. A prior test-retest examination in 104 maintenance patients by two interviewers (1-week interval) revealed low to moderate ICC values (.38-.52), for specific EuropASI domains but acceptable test-retest reliability (ICC: .68) for the total score. Because of the restricted reliability only the total score was used in some confounder analyses as a measure for overall severity.

\subsubsection{Urine drug screens. All patients underwent standardized urine drug screenings} supervised by a nurse at baseline and at all follow-ups. Drug screening tools were provided by the study center (Drug screen Multi 7; von Minden GmbH, Germany). The screeningwas performed for methadone, buprenorphine, other opiates, cocaine, amphetamines, methamphetamines, benzodiazepines and cannabis.

\subsection{Measures}

Retention was defined as the number of weeks that elapsed between study entry and the last week in treatment assessed at 3-month intervals. Retention rates were calculated for the total sample without those that had become abstinent. Mortality was defined as any death occurring during the follow-up period and after study entry, irrespective of the cause of death. 
Abstinence/abstinence treatment was defined as successful discontinuation of maintenance treatment by the doctor, either because the patient was considered to be clean (no positive opiate urine screens for 4 weeks) or because of change to abstinence treatments. Information about abstinence was based on the treating physician's rating in the follow-up interviews and a negative urine test. Additionally, separate telephone interviews were conducted with these doctors 2-4 months later to confirm whether patients were still abstinent. Only patients who were confirmed to be still abstinent in this second interview were counted as abstinent. Concomitant drug use was defined as a positive urine sample on the day of the personal examination (baseline and follow-ups). Any positive screening for unprescribed opiates (methadone, buprenorphine, other such as codeine), cannabis, cocaine, methamphetamine, amphetamine, benzodiazepine, and hallucinogens was counted. Presence of methadone in buprenorphine patients was counted as concomitant drug use and vice versa. Somatic health and mental health was defined as the presence of clinician-rated ICD-10 diagnoses of 9 explicitly described groups of somatic disorders (e.g. cardiovascular conditions, liver diseases) with an open entry question, and 12 groups of mental disorders. Doctors were encouraged to code all applicable diagnoses definitely present during the past 12 months. In addition, the Brief Symptom Inventory (BSI) total score was used to evaluate changes between baseline and follow-up in past week self-reported psychopathology.

\subsection{Statistical procedures}

Except for retention, mortality and abstinence from baseline to follow-up, comparisons were made for the total sample as well as for settings groups. Dimensional and binary measures were tested for follow-up versus baseline differences using aWald F-test which adjusted for design effects and clustering within primary care units by applying the Huber-White sandwich matrix (Royall, 1986). For group differences, the follow-up status was regressed on group status while adjusting for the baseline status.

For binary, multicategorial and dimensional outcomes, logistic, multinomial logistic and linear regressions were used, respectively, while again applying the sandwich method (Royall, 1986). Retention rate curves over the follow-up period were estimated with the Kaplan-Meier method, adjusting for overall mean substitution time differences in settings before baseline using Cox regression (Therneau and Grambsch, 2000). When considering differences in primary outcomes between small, medium and large primary care settings, design effects, clustering of observation, duration of maintenance treatment and, if applicable, type of medication prior to baseline were also statistically adjusted. It should be noted that in 21 patients medication transfer occurred during the follow-up period. In these cases patients were assigned to the medication group in which they had spent most of the follow-up period.

\section{Results}

\subsection{Socio-demographic and selected clinical characteristics of the study sample}

Table 2 presents the baseline demographics and selected structured clinical interview information for the total sample and by setting. Patients in all three groups are rather similar in most of the measured variables, except for lower rates of patients in SSC that were employed or treated with buprenorphine. Patients in SSC also revealed significantly lower pre-baseline treatment duration and a somewhat higher EuropASI severity score at baseline as compared to PMC and medium settings.

\subsection{Retention rates and time to drop out}


Fig. 2a-c show the retention rates from baseline to 1-year follow-up, excluding $n=274$ patients that terminated the maintenance program because of either abstinence or referral to abstinence programs. Adjusted for differences between groups with regard to prior treatment duration, both medication groups reveal a steady decline of retention over the 52 weeks observation time of about 25\%. Fig. 2c reveals that retention during the follow-up period is associated with length of previous retention in the treatment before baseline. Patients who had just started their current maintenance therapy in the 4 weeks prior to baseline, irrespective of type of medication, had the lowest retention rate as compared to patients with $>6$ month (82\%) prior treatment (61\%, HR: 2.7; 95\% CI: 2.1-4.4), followed by patients with 2-6 months prior treatment (70\%, HR: 2.0 95\% CI: 1.6-2.4). Fig. 2b reveals better long-term retention in PMC settings as compared to SSC (HR: 1.4, 95\% CI: 1.1-1.7) largely due to changes after week 42 . Reasons for not retaining patients in therapy were similar in most subgroups examined, except for the methadone group, for which more patients were not retained because of imprisonment (HR: 1.7; 95\% CI: 1.3-2.5).

\subsection{Discontinuation, death and abstinence in the total sample and by provider setting}

Overall, at the end of the 1-year observation period, $21.6 \%$ of the total baseline sample ( $\mathrm{n}=$ 2442 patients) were not retained in treatment. In $4.1 \%$ of the sample treatment was terminated because patients were rated as being abstinent, $7 \%$ had changed to drug-free treatments; the overall mortality rate was $1.1 \%$. Among clinical reasons for discontinuation of maintenance treatment "serious concomitant drug use" was the most frequent. Only 9 out of the total number deaths occurred during the treatment itself, 22 happened in patients that either discontinued treatment on their own or after treatmentwas discontinued because of disciplinary reasons (severe and continued concomitant drug use).

\subsection{Rates of non-retention, death, and abstinence overall and by setting $(n=2442)$}

SSC had higher rates of non-retention (OR: 1.44; 95\% CI: 1.05-1.94), lower rates of abstinent patients (OR: 0.55; 95\% CI.0.3-0.99), but also higher rates of patients changing to abstinence treatments (OR: 2.01; 95\% CI: 1.19-3.33). These differences remained significant even after adjusting for duration of current treatment. SSC appear to have lower mortality rates as compared PMC (Fig. 3).

\subsection{Changes in concomitant drug use, somatic and mental health}

Table 3 reports the baseline and follow-up findings among those still in treatment $(n=1615)$ by type of setting, while controlling for baseline values and accounting for clustering of observations within settings.

\subsection{Concomitant drug use}

As evidenced by positive drug screens on the day of the assessments, $19.3 \%$ of all patients screened positive for non-prescription opiate use and $48.9 \%$ for use of other nonprescribed drugs at baseline. Overall, concomitant drug use rates dropped significantly at follow-up (15.8\%, F: 4.4, p <.05 and 46.3\%, F: 7.0, p < .01, respectively). Neither the rate of patients with at least one positive screen (54.9 and 52.5\%) nor the number of positive screens per patient was significantly reduced. Since one could assume, that most changes had taken place in the first weeks or months of treatment, we separately analyzed the subset of 352 patients that started the treatment within the month before the start of the study and found that this 
subgroup had no noteworthy different outcome patterns as opposed to those with longer treatment. Except for higher rates of screen-negatives in small PMC settings, there were no differences by type of setting or by type of maintenance drug. A closer examination of the type of concomitant drug use at follow-up (Fig. 4) revealed that patients in large specialized centers, as opposed to small PMC settings, had considerably higher concomitant drug use rates of cannabis (OR: $1.9(1.3-2.8)$ ), benzodiazepines (OR: $1.7(1.0-2.8)$ ) and any opiates (OR: 1.7 (1.0-2.9)) even after adjusting for prior treatment duration differences.

\subsection{Somatic and mental health}

At baseline, patients overall and all setting groups reveal a considerable degree of somatic morbidities. Only a few patients had "no" diagnosis and the average number of somatic diagnoses coded by the doctor were 3.4. Most frequent groups of diagnoses were HCV infection (67.1\%), pulmonary diseases (23.2) and cardiovascular diseases (15.1\%); 7.3\% of patients were diagnosed as having HIV-infection/AIDS. At follow-up, there was a noteworthy and significant reduction for almost all disorders, except for HIV/AIDS, with no notable differences by type of setting.

At baseline, all patients also had at least one 12-month mental disorder; most frequent diagnoses were depressive and personality disorders. However, although there was modest significant reduction in the number of patients with at least one 12-month mental disorder (100\% vs. $82.9 \%)$ at follow-up, it should be noted that overall the number of diagnoses/patient increased $(1.7-2.2, \mathrm{~F}=42.4)$, due to a higher frequency of coded sleep and stress disorders. Unlike the broader 12-month time window for physicians-rated mental disorders, the cross-sectional symptom self-report on the Brief Symptom Inventory (BSI) revealed a modest, though significant improvement from baseline to follow-up.

\section{Discussion}

The findings from this nationally representative sample of opioid-dependent patients in various types of maintenance treatments underline the overall effectiveness of various forms and provider formats of agonist maintenance treatments with methadone and buprenorphine. In line with previous findings from clinical studies (Merrill, 2002; Wittchen et al., 2005; Connock et al., 2007), our data covering a longer time period than most previous studies, demonstrate low mortality rates of about $1 \%$, a relatively high 12-month retention in treatment of about $70 \%$ and statistically significant, albeit modest improvements in most of the 12 months outcome domains considered. In a small proportion (11\%) of patients, routine maintenance treatments also seem to reach either complete abstinence (4\%) or to subsequently motivate the patient to enter an abstinence psychosocial treatment (7\%). These outcomes are considered as explicit goals by the German maintenance treatment regulations. Considering the long illness history and chronicity of on average over 14 years and the remarkably poor somatic and mental health status of patients, these overall findings are encouraging. It should be noted though, that in this prevalence sample, patients who just started the treatment within the previ-ous month had somewhat lower retention rates (61\%) than those that were in treatment for 6 months or more (82\%), suggesting that in an incidence sample of newly admitted patients retention rates would be slightly lower than the reported $70 \%$ in this study.

Beyond this demonstration of overall effectiveness, the core noteworthy finding of the study is that the outcome of patients treated in small-scale primary care settings, characterized by lesser degree of expertise and a lack of psychosocial intervention resources "in house", 
appears to be at least equal to large-scale specialized substitution centers with a considerable degree of additional medical and psychosocial resources. This finding, however, should be taken with caution, because as a naturalistic study and considering the level of detail, we were not able to comprehensively control for all potential confounders. In line with related findings of our study and recent reviews (Connock et al., 2007) though, this finding indicates that the implementation of maintenance treatments in primary care settings is a feasible, promising and potentially cost-effective option (Gossop et al., 2003; Merrill, 2002; Hutchinson et al., 2000). A further important public health implication is that this strategy might also improve access to maintenance treatments in underserved areas.

The strengths of the study are the reliance on a large nationally representative and heterogeneous sample of maintenance treatment providers and a random sample of their patients, the length of the observation period, and the coverage of a wide range of variables describing course and outcome, including urine drug tests.

There are also some important limitations. Firstly, this is a strictly observational, naturalistic study and not a randomized controlled trial. Thus, causal inferences are not possible and all causal suggestions regarding the effects of different treatments and settings need to be interpreted with caution. One might even argue that the use of any formal testing, such as the use of odds ratios and hazard rates in combination with confounder analyses might be inappropriate, because of the risk of being misinterpreted as being suggestive for causal relationships. Therefore, this paper almost exclusively uses such measures of association the way they were originally designed for in descriptive epidemiology. Further limitations are: (i) only 59\% of eligible settings participated, and no information is available regarding the question whether non-participating settings differ from those that participated; also "only" $72 \%$ of the eligible patients participated in the study and not all of these patients provided 12month data. (ii) Patients not speaking German fluently were excluded from the study because of the assessment instruments. One might speculate that those patients are typically more severe and have a worse prognosis. (iii) Objective measures of alcohol use and dosing of maintenance drugs were not considered. (iv) Further, one needs to take into account that psychometric data for some of the measures are of limited reliability and in some instances no validity data are available. This limitation might be particularly relevant for the use of the EuropASI as a measure for potential confounders.

Taking into account these limitations, there are several encouraging observations:

(1) The retention rate in routine care is relatively high and resembles findings in controlled clinical trials with incident treatment samples (Wittchen et al., 2005; Connock et al., 2007), if one accounts for the 12-month duration of the study. The good 12-month retention rate also applies to new onset treatment cases (within 4 weeks before baseline). Further - and despite of the finding that new onset MT patients seem to perform slightly better than BMT patients no remarkable difference in retention between MT and BMT was found as suggested by some studies (Connock et al., 2007), if controlled for length of prior maintenance on the respective drug. The high retention is particularly noteworthy, because of arguments not to integrate expensive long-term somatic treatments (e.g. HCV treatment) to maintenance programs because of assumed poor adherence, clinical and cost considerations (Schaefer et al., 2004). The finding that more than $65 \%$ of randomly selected maintenance patients were retained over 12-month does not substantiate this reservation (Schäfer et al., 2005). One might speculate that the retention rate in our study may be largely due to the higher degree of permissiveness of treating physicians. As evidenced by the substantial rates of urine screen confirmed illicit drug use of $20 \%$ opiates and almost $50 \%$ of other drugs at baseline and follow-up, the 
treatment of patients with serious concomitant drug use was obviously not discontinued in at least one fifth of the sample. This is in contrast to German guidelines of good clinical practice that recommends the exclusion of such patients from further maintenance treatment. However, this does not account entirely for the finding that PMCs seem to have higher retention. Additional analyses do not reveal that discontinuation rates due to disciplinary reasons in SSC and medium centers among concomitant drug users are different from the ones observed for PMC. Further evaluative activities suggest that patients in smaller settings might have a higher adherence to the doctor.

(2) Concomitant drug use is apparently not only the major reason for discontinuation of treatment, but also a frequent complication of the clinical course. The data suggest that this might be particularly the case for the patients treated in SSC that show higher rates of concomitant cannabis, benzodiazepine, opiate and cocaine use at follow-up. Smaller PMC appear to have considerably lower rates of concomitant drug use, even after accounting for some baseline differences. The finding of higher concomitant drug use rates in patients treated in SSCs compared to those treated in smaller PMCs, requires caution and further examination, due to the fact that this is not a randomized study. We cannot exclude the possibility that in the current study with its naturalistic design, patients in small PMCs and large centers differ in measures that were not taken into account. Further analyses by type of substance will examine to what degree there is an interaction with type of substitution drug, as we will deal with special subpopulations as that might explain the difference. Overall, BMT patients show lower concomitant use rates. However, it is troubling that all types of settings have only limited success in reducing concomitant drug use rates (Gossop et al., 2003).

(3) Maintenance patients in routine care are characterized by an extremely high burden of somatic and mental disorders. Almost all MT patients in our study reveal a considerable degree of somatic and mental disorder morbidities and could be described as multimorbid. Over 7\% were HIV positive or had AIDS, 67\% were HCV positive, 23 and 15\%, respectively had severe cardiovascular or pulmonary diseases, and the majority of patients suffered from a depressive disorder. It is against this impressive spectrum of morbidity that the observed changes in somatic health in the follow-up period should be appraised. Overall, all types of settings were successful in reducing somatic morbidities and improving the somatic health status. In contrast, there was only marginal improvement in patients' mental health; rates for sleep disorders, PTSD and acute stress disorders even increased, Taking into account that many settings offer a considerable spectrum of mental health treatment resources (i.e. clinical and psychological and social treatment components involving psychiatrists and psychologists), the failure to reduce mental disorders is remarkable and needs further clarification. The fact that all types of settings failed to attain substantial improvements in this domain seems to suggest that the treatment of mental disorders in maintenance patients is deficient or at least not sufficiently effective.

To summarize, the present data and findings suggest that in the German health care system maintenance treatment in a PMC setting (small and medium) is a promising treatment option for patients with opioid dependence with potential good outcomes in terms of retention and health promotion.

The findings regarding possible differential outcomes by type of setting need further examination and replication, preferably in randomized trials. The current findings from this observational study might have important implications though in terms of allocation as well as health economic considerations. The finding suggesting that smaller PMC settings perform as good as SSCs - if it could be confirmed in more detailed analyses - might have important 
implications from a public health perspective, such as making maintenance treatments more cost-effective and more easily available in rural and non-metropolitan areas. The results may also provide a first step in shaping the treatment system for opioid addicts. Given better retention in PMC, the future use of SSC may be optimized by focusing more on handling crisis situations and problematic patients failing in the routine system.

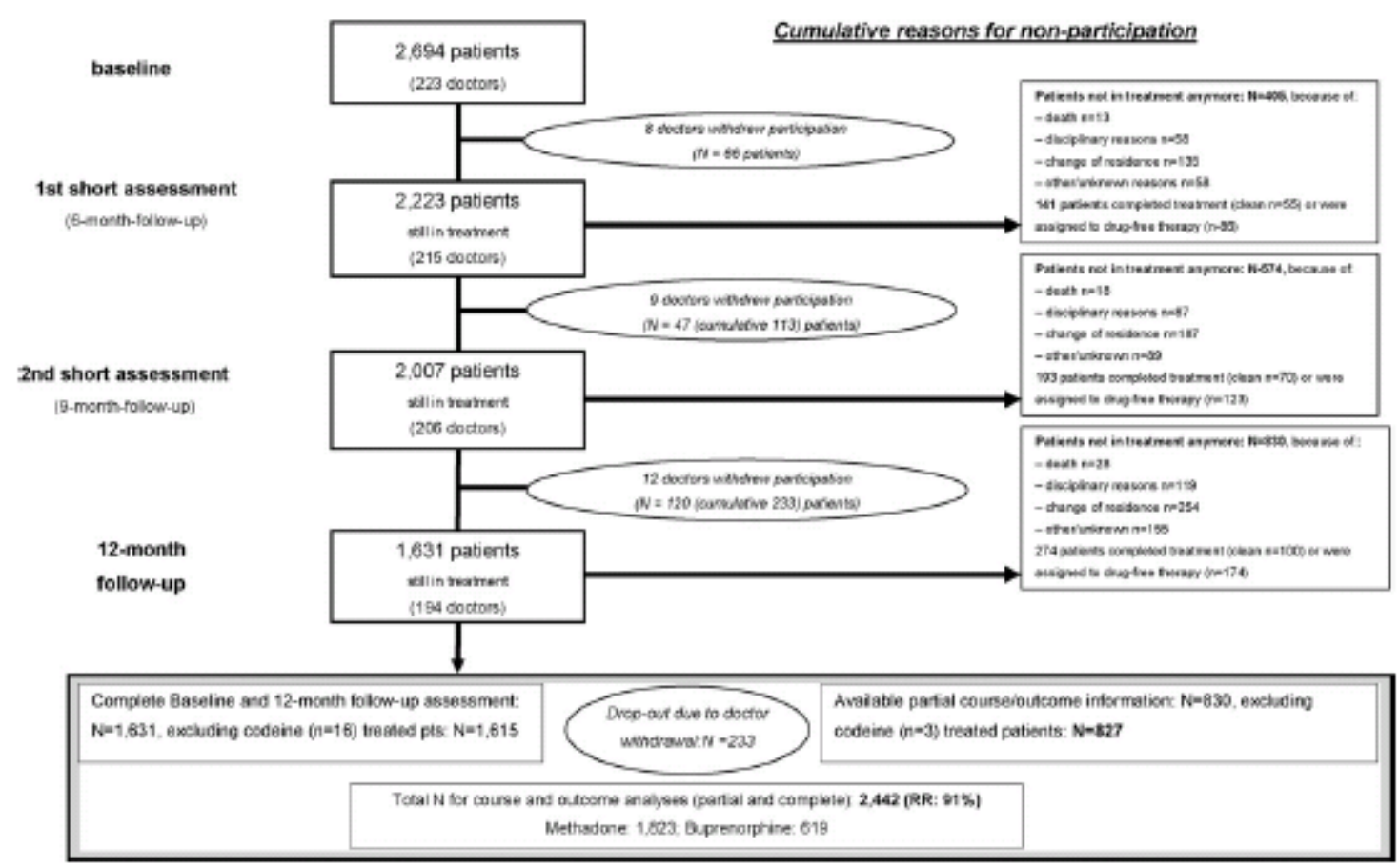

Fig. 1. Flow diagram of patients during the follow-up period. 

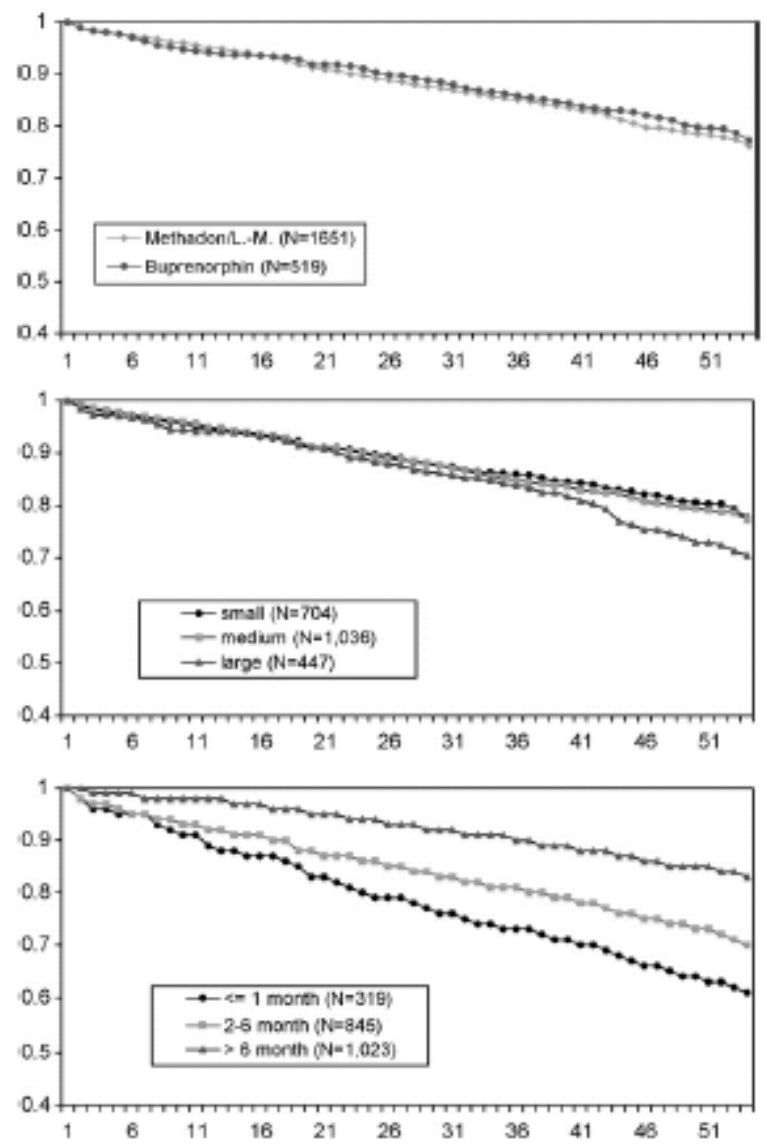

Fig. 2. a-c 12-Month retention rates by type of medication (upper panel), and provider setting (middle panel) and length of treatment (lower panel) across weeks of the observation period.

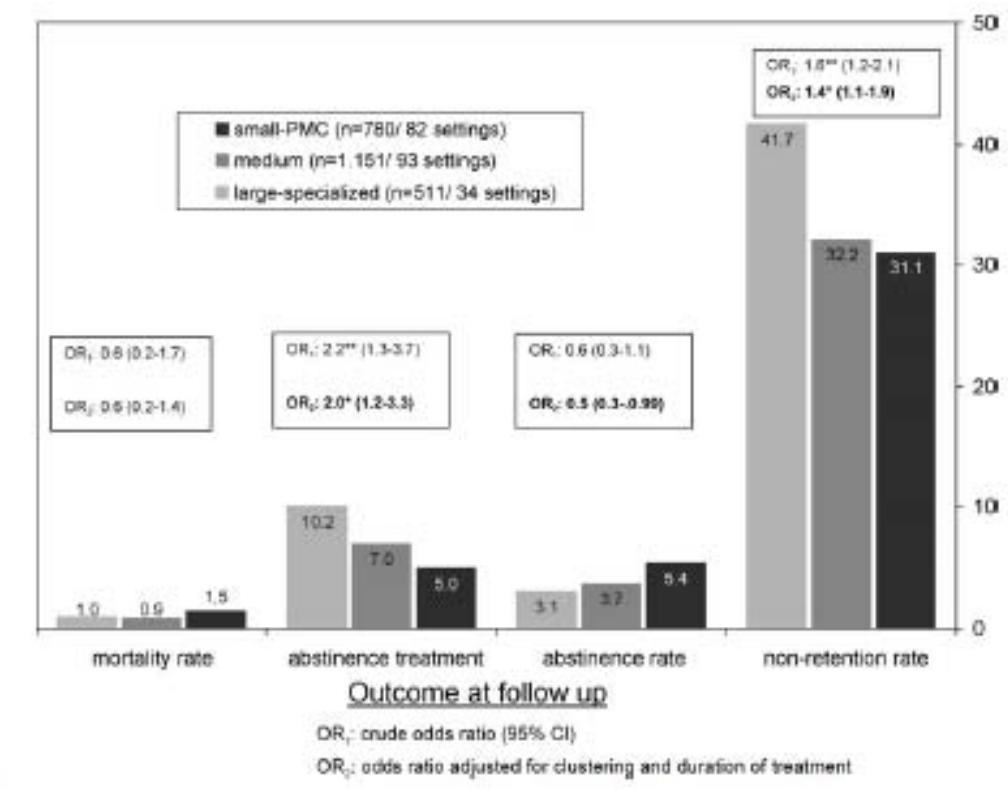

Fig. 3. Rates (percentage of patients) of non-retention, death, and abstinence overall and by setting ( $N=2442$ ). 


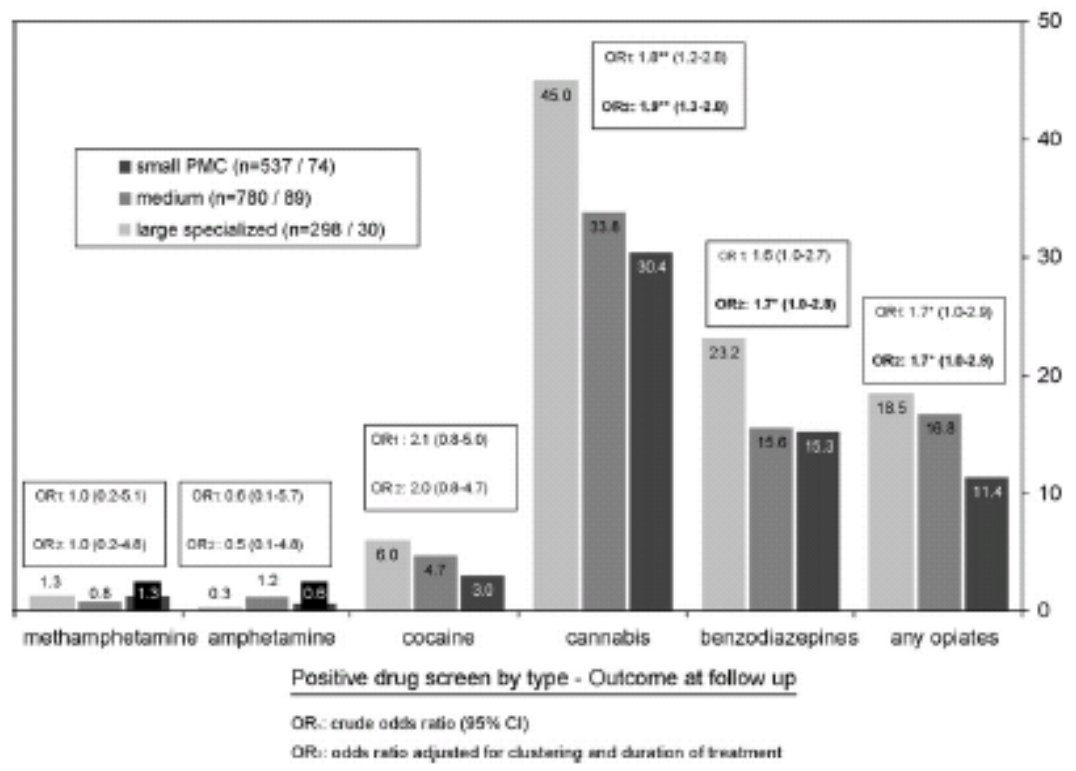

Fig. 4. Concomitant drug use (percentage of patients) by provider setting.

Table 1

Selected provider characteristics of small PMC, medium and large-scale SSC settings

\begin{tabular}{|c|c|c|c|}
\hline & Type of setting: Small $(n=86)$ & Type of setting: Medium $(n=101)$ & Type of setting: Large (36), \\
\hline \% family doctor & $79.1 \%$ & $82.3 \%$ & $45.7 \%$ \\
\hline \% other & $16.9 \%$ & 0 & $22.9 \%$ \\
\hline \multicolumn{4}{|l|}{ Psychosocial interventions (current) } \\
\hline Within setting & $8.5 \%$ & $48.6 \%$ & $81.3 \%$ \\
\hline Number of current pts (mean/S.D.) & $5.1(2.3)$ & $26.4(9.7)$ & $118.2(24.6)$ \\
\hline$\%$ pts with urine test weekly & $61.1 \%$ & $57.4 \%$ & $48.0 \%$ \\
\hline \% pts with take-home prescription & $75.4 \%$ & $57.5 \%$ & $33.2 \%$ \\
\hline Average no. of days take-home & $2.1(0.4)$ & $1.9(0.6)$ & $1.7(0.6)$ \\
\hline Face-to-face contact/week (mean/S.D.) & $3.7(2.3)$ & $4.9(2.3)$ & $5.6(2.1)$ \\
\hline Average duration of contact in minutes (mean/S.D.) & $10.9(7.4)$ & $9.9(6.8)$ & $10.9(7.6)$ \\
\hline
\end{tabular}


Sodio-denognphic and selociat clinical charatieristios of the study sanple

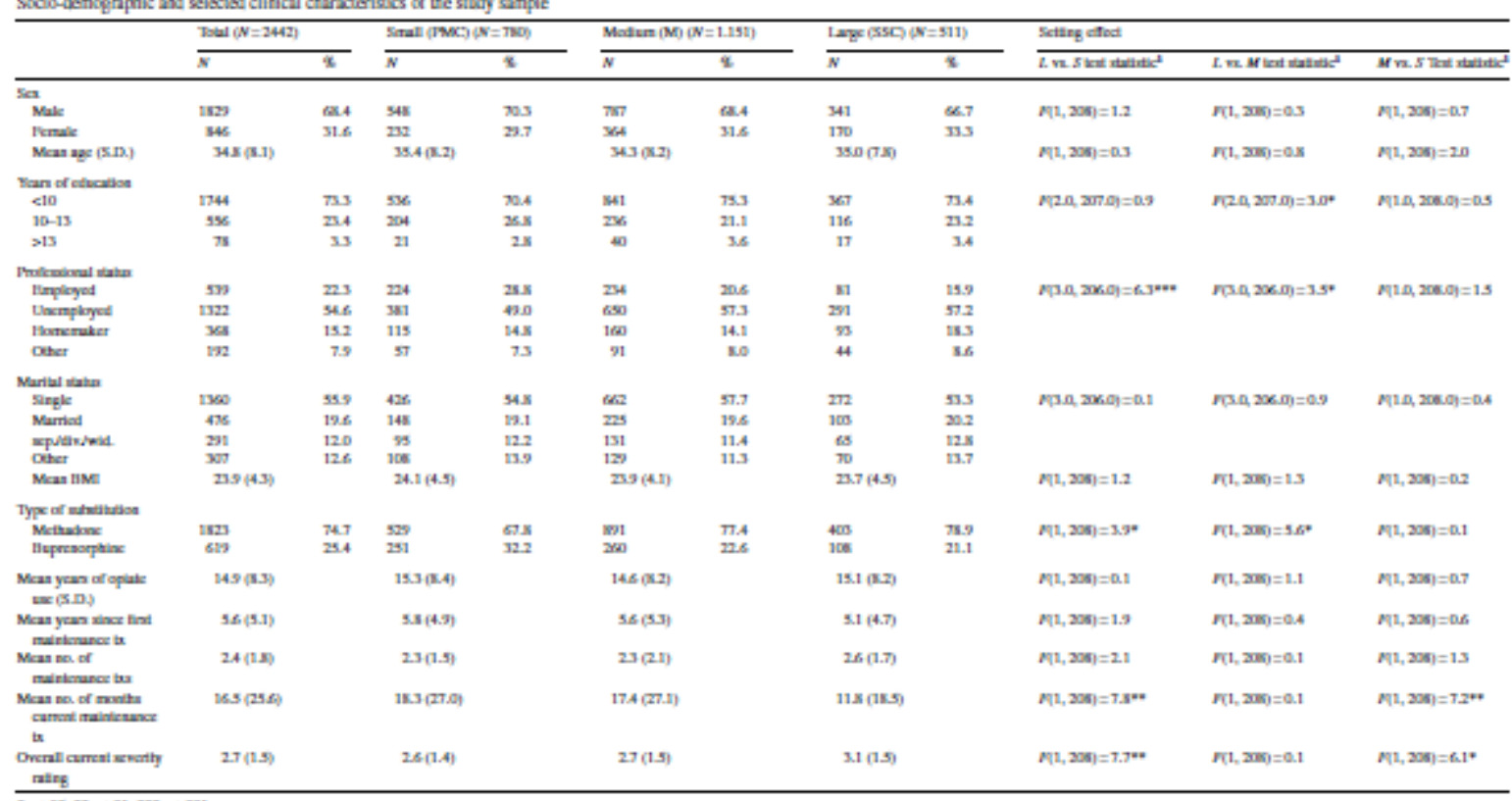

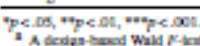

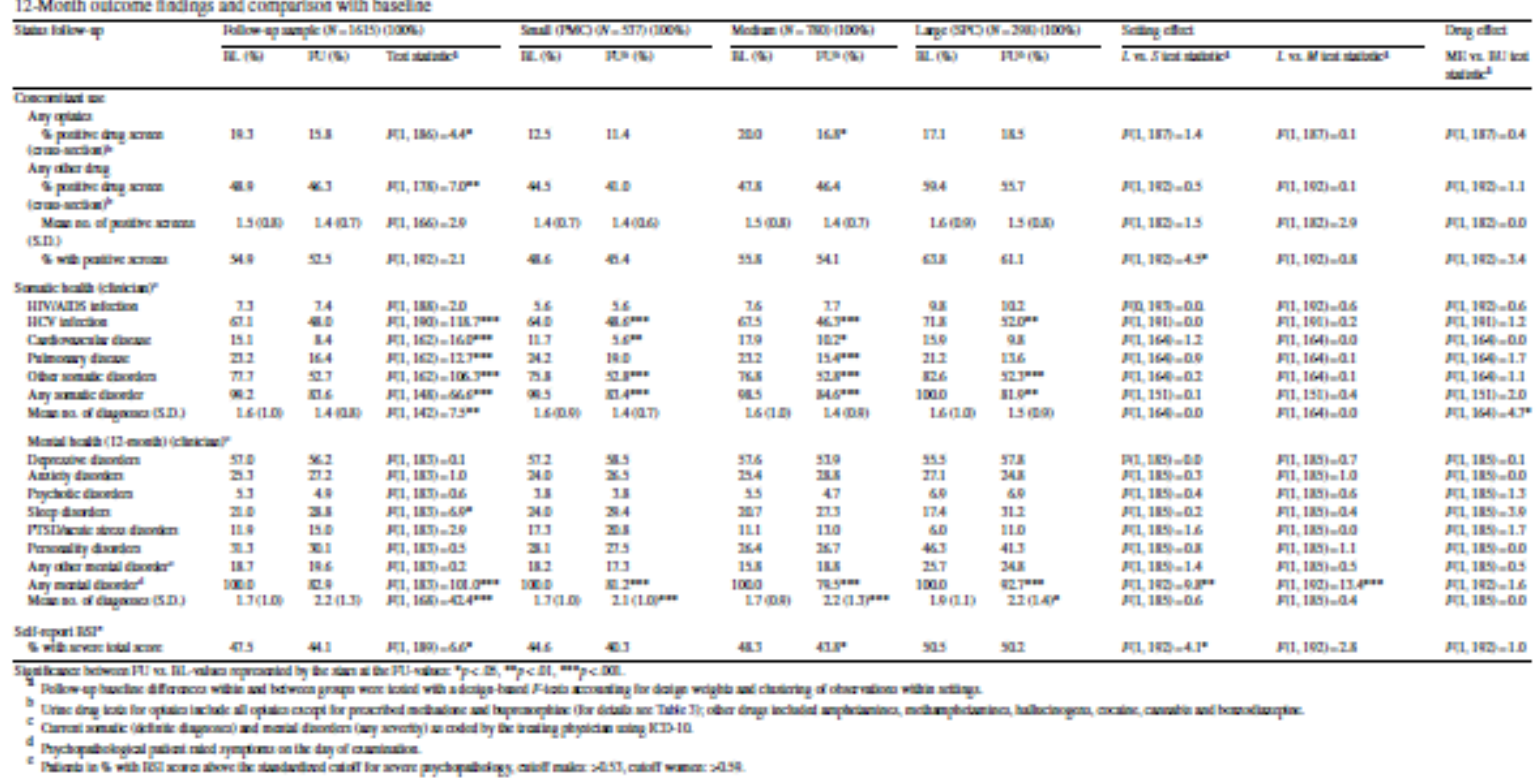

\section{Conflict of interest}

Gerhard Bühringer, J. Rehm and Jens Siegert declare they have no conflicts of interest. Dr. Wittchen has a financial interest/arrangement or affiliation with one ore more organizations that could be perceived as a real or apparent conflict of interest in the context of the subject of this presentation. He receives or has in the past 3 years received research support from: Eli Lilly and Company; Novartis; Pfizer; Schering-Plough. He is currently or in the past 3 years has been a consult for: Eli Lilly; GlaxoSmithKline Pharmaceuticals; Hoffmann-La Roche Pharmaceuticals; Novartis; Pfizer; Wyeth. He receives or has in the past 3 years received speaking honoraria from: Novartis; Schering-Plough; Pfizer; Wyeth. Sabine M. Apelt has a financial interest/arrangement or affiliation with one or more organizations that could be perceived as a real or apparent conflict of interest in the context of the 
subject of this presentation. She receives or has in the past 3 years received honoraria from: Essex pharma GmbH. Prof. Soyka has worked as a consultant for Sanofi Aventis, Essex Pharma, Alkermes, Lipha, Forrest Lab, Bayer. Dr. Gastpar has been during the last 3 years member of consultation boards for Astra-Zeneca, GlaxoSmithKline, Lundbeck, Pfizer, Servier and Wyeth Germany and has received speaking honoraria from Schwabe, Servier and Zeller Pharmaceuticals. Dr. Backmund has a financial interest/arrangement or affiliation with one ore more organizations that could be perceived as a real or apparent conflict of interest in the context of the subject of this presentation. He receives or has in the past 3 years received research support from: Schering-Plough. He is currently or in the past 3 years has been a consult for: Essex pharma GmbH. He receives or has in the past 3 years received speaking honoraria from: Schering-Plough; Essex pharma GmbH, Roche Pharma AG, Sanofi-Aventis Deutschland GmbH, Bristol-Myers Squibb.

Dr. Gölz has no financial relations to the companies that produce the substances that were used in the substitution treatment under consideration. Dr. Tretter has no financial interest/ arrangement or affiliation with one or more organizations that could be perceived as a real or apparent conflict of interest in the context of the subject of this presentation. In the past 3 years, he has received a speaking honoraria from: Mundi Pharma. Dr. Schäfer has a financial interest/arrangement or affiliation with one or more organizations that could be perceived as a real or apparent conflict of interest in the context of the subject of this presentation. He receives or has in the past 3 years received speaking honoraria from: Pfizer; Astra-Zeneka, UCB, Schering-Plough, Hoffmann La-Roche. He also received Research Support from: Lundbeck; Pfizer; Schering-Plough, Hoffmann La-Roche, UCB. Prof. Scherbaum has a financial interest/arrangement or affiliation with one ore more organizations that could be perceived as a real or apparent conflict of interest in the context of the subject of this presentation. He receives or has in the past 3 years received research support from: Aventis-Pharma. Dr. M.R. Kraus has a financial interest/ arrangement or affiliation with one or more organizations that could be perceived as a real or apparent conflict of interest in the context of the subject of this presentation. He receives or has in the past 3 years received research support from: Schering- Plough (essex pharma, Germany). He is currently or in the past 3 years has been a Consult for: Schering-Plough (essex pharma, Germany). He receives or has in the past 3 years received Speaking Honoraria from: Schering-Plough (essex pharma, Germany).

\section{Acknowledgements}

This study is part of the collaborative COBRA study (Cost-Benefit and Risk Appraisal of Substitution Treatments, www.cobra-projekt.de), supported by the German Federal Ministry of Research and Technology (BMBF No. 01EB0440- 0441/01EB0142) as part of the Addiction Research Network "ASAT" (www.asat-verbund.de). The field work and health economic program components were further supported by an unrestricted educational grant from essex pharma GmbH, Germany. We wish to thank all doctors and staff members of the participating clinics for their continued support and collaboration. Contributors. Hans-Ulrich Wittchen has planned the investigation and written the manuscript. Sabine M. Apelt provided substantial contribution to the writing of the manuscript. Dr. Gölz has participated in the recruitment of study participants and has provided counselling for the conceptualisation of the study questionnaires. Dr. Tretter provided substantial contribution to the writing of the manuscript, the interpretation, and the discussion of the results. Jens Siegert has planned and conducted the statistical analysis. Jürgen Rehm has provided substantial comments on the writing of the manuscript as well as consulting for the statistical analysis and its presentation. Gerhard Bühringer provided substantial contribution to the writing of the manuscript. Prof. Scherbaumwas responsible for carrying out the study in one of the participating centers. He provided substantial contribution to the writing of the manuscript. Dr. Schäfer and Profs. Kraus, Gastpar, and Soyka provided supervision of and substantial contribution to the writing of the manuscript.

\section{References}

- Connock, M., Juarez-Garcia, A., Jowett, S., Frew, E., Liu, Z., Taylor, R.J., Fry-Smith, A., Day, E., Lintzeris, N., Roberts, T., Burls, A., Taylor, R.S., 2007. Methadone and 
buprenorphine for the management of opioid dependence: a systematic review and economic evaluation. Health Technol. Assess. 11 (9), 1-171.

- Farell, M., Howes, S., Verster, A., Davoli, M., Solberg, U., Greenwood, G., Robertson, K. (Eds.), 2000. Reviewing Current Practice in Drug-substitution Treatment in the European Union. Insights 3. MCDDA Series. Office for Official Publications of the European Community, Luxembourg.

- Gerra, G., Borella, F., Zaimovic, A., Moi, G., Bussandri, M., Bubici, C., Bertacca, S., 2004. Buprenorphine versus methadone for opioid dependence: predictor variables for treatment outcome. Drug Alcohol Depend. 75, 37-45.

- Gossop, M., Marsden, J., Stewart, D., Treacy, S., 2001. Outcomes after methadone maintenance and methadone reduction treatments: two-year follow-up results from the National Treatment Outcome Research Study. Drug Alcohol Depend. 6, 255-264.

- Gossop, M., Stewart, D., Browne, N., Marsden, J., 2003. Methadone treatment for opiate dependent patients in general practice and specialist clinic settings: outcomes at 2-year followup. J. Subst. Abuse Treat. 24, 313-321.

- Greiner, W., Weijnen, T., Nieuwenhuizen, M., Oppe, S., Badia, X., Busschbach, J., Buxton, M., Dolan, P., Kind, P., Krabbe, P., Ohinmaa, A., Parkin, D., Roset, M., Sintonen, H., Tsuchiya, A., de Charro, F., 2003.Asingle European currency for EQ-5D health states. Results from a six-country study. Eur. J. Health Econom. 4 (3), 222-231.

- Groß, A., Soyka, M., 1999. Buprenorphine-a new approach to pharmacotherapy of opiateaddicted patients. Suchtmed 1, 5-14.

- Gsellhofer, B., Küfner, H., Vogt, M., Weller, D., 1999. European Addiction Severity IndexEuropASI. Manual for Training and Execution. Schneider Verlag, Stuttgart.

- Guy, W., 1976. Clinical global impressions. In: National Institute of Mental Health (Ed.), ECDEU Assessment for Psychopharmacology, 1st ed. National Institute of Mental Health, Rockville, USA, pp. 21-27.

- Hutchinson, S.J., Taylor, A., Gruer, L., Barr, C., Mills, C., Elliott, L., Goldberg, D.J., Scott, R., Gilchrist, G., 2000. One-year follow-up of opiate injectors treated with oral methadone in a GP-centred programme. Addiction 95 (7), 1055-1068.

- Johnson, R.E., Jaffe, J.H., Fudala, P.J., 1992. A controlled trial of buprenorphine treatment for opioid dependence. JAMA 267, 2750-2755.

- Kakko, J., Svanborg, K.D., Kreek, M.J., Heilig, M., 2003. 1-Year retention and social function after buprenorphine-assisted relapse prevention treatment for heroin dependence in Sweden: a randomised, placebo-controlled trial. Lancet 361, 662-668.

- $\quad$ King, V.L., Stoller, K.B., Hayes, M., Umbricht, A., Currens, M., Kidorf, M.S., Carter, J.A., Schwartz, R., Brooner, R.K., 2002. A multicenter randomized evaluation of methadone medical maintenance. Drug Alcohol Depend. 65,137-148.

- Kintzel, M., 2007. Charakteristika von Substitutionseinrichtungen in Deutschland: Ein Vergleich verschiedener Versorgungstypen. Unpublished Diploma Thesis, TUD.

- Law, F.D., Nutt, D.J., 2003. Maintenance buprenorphine for opioid users. Lancet 361 (9358), 634-635.

- Layson-Wolf, C., Goode, J.V., Small, R.E., 2002. Clinical use of methadone. J Pain Palliat. Care Pharmacother. 16 (1), 29-59.

- $\quad$ Ling, W., Charuvastra, C., Kaim, S.C., Klett, C.J., 1976. Methadyl acetate and methadone as maintenance treatments for heroin addicts. A veterans administration cooperative study. Arch. Gen. Psychiatry 33 (6), 709-720.

- $\quad$ Ling, W., Wesson, D.-R., 2003. Clinical efficacy of buprenorphine: comparisons to methadone and placebo. Drug Alcohol Depend. 70 (Suppl. 1), 49-57.

- Mattick, R.P., Ali, R., White, J.M., O’Brien, S., Wolk, S., Danz, C., 2003. Buprenorphine versus methadone maintenance therapy: a randomized double-blind trial with 405 opioiddependent patients. Addiction 98 (4),441-452.

- $\quad$ Mattick, R.P., Breen, C., Kimber, J., Davioli, M., 2004. Methadone maintenance therapy versus no opioid t replacement therapy for opioid dependence. The Cochrane Library, vol. 4. John Wiley and Sons, Chichester, UK (Cochrane Review). 
- McLellan, A.G., Arndt, I.O., Metzger, D.S., Woody, G.E., O’Brien, C.P., 1993. The effects of psychosocial services in substance abuse treatment. JAMA 269, 1953-1959.

- Merrill, J.O., 2002. Policy progress for physician treatment of opiate addiction. J. Gen. Intern. Med. 17, 361-368.

- Mintzer, M.-Z., Stitzer, M.-L., 2002. Cognitive impairment in methadone maintenance patients. Drug Alcohol Depend. 67 (1), 41-51.

- Poser, S., Poser, W., 1996. Medikamente-Missbrauch und Abhängigkeit: Entstehung. Verlauf. Behandlung. Thieme, Stuttgart.

- Royall, R.M., 1986. Model robust confidence intervals using maximum likelihood estimators. Statist. Rev. 54, 221-226.

- Salsitz, E.A., Joseph, H., Frank, B., Perez, J., Richman, B.L., Salomon, N., Kalin, M.F., Novick, D.M., 2000. Methadone medical maintenance (MMM): treating chronic opioid dependence in private medical practice-a summary report (1983-1998). Mt. Sinai J. Med. 67, 388-397.

- Schaefer, M., Heinz, A., Backmund, M., 2004. Treatment of chronic hepatitis C in patients with drug dependence: time to change the rules? Addiction 99 (9), 1167-1175.

- Schäfer, A., Scheurlen, M., Felten, M., Kraus, M.R., 2005. Physician-patient relationship and disclosure behaviour in chronic hepatitis $\mathrm{C}$ in a group of German outpatients. Eur. J. Gastroenterol. Hepatol. 17, 1387-1394.

- Soyka, M., Banzer, K., Buchberger, R., Völkl, M., Naber, D., 1997. Methadone substitution in opioid dependence. Nervenheilkunde 16, 347-352.

- Soyka, M., Fischer, G., Ladewig, D., Blanke, J., Nowak, M., Poehlke, T., Penning, R., Liess, H., Schütz, C., Küfner, H., 2000. Recommendations for buprenorphine (SUBUTEX) substitution therapy for opioid-dependent patients in Switzerland Austria and Germany (consensus reports). Suchtmed 2 (1), 43-53.

- Strain, E.C., Spitzer, M.L., Liebson, I.A., Bigelow, G.E., 1994. Comparison of buprenorphine and methadone in the treatment of opioid dependence. Am. J. Psychiatr. 151 (7), 1025-1030.

- Therneau, T.M., Grambsch, P.M., 2000. Modeling Survival Data-Extending the Cox Model. Springer, New York.

- Vollmer, H.-C., Krauth, J., 2001. Therapie der Drogenabhängigkeit. In: Tretter, F., Müller, A. (Eds.), Psychologische Therapie der Sucht. Hogrefe, Göttingen, pp. 395-438.

- Waal, H., Haga, E., 2003. Maintenance Treatment of Heroin Addiction: Evidence at the Crossroads. Cappelen Akademisk Forlag, Oslo.

- Walsh, S.L., Gilson, S.L., Jasinski, D.R., Stapleton, J.M., Phillips, R.L., Dannals, R.F., Schmidt, J., Preston, K.L., Grayson, R., Bigelow, G.E., Sullivan, J.T., Contoreggi, C., London, E.D., 1994. Buprenorphine reduces cerebral glucose metabolism in polydrug abusers. Neuropsychopharmacology 10, 157-170.

- Wittchen, H.U., Apelt, S., Bühringer, G., Gastpar, M., Backmund, M., Gölz, J., Kraus, M., Tretter, F., Klotsche, J., Siegert, J., Pittrow, D., Soyka, M., 2005. Buprenorpione and methadone in the treatment of opioid dependence. Methods and design of the COBRA study. Int. J. Methods Psychiatr. Res. 14 (1), 14-28.

- Wittchen, H.U., Lachner, G., Wunderlich, U., Pfister, H., 1998. Test-retest reliability of the computerized DSM-IV Version of the Munich-Composite International Diagnostic Interview(M-CIDI). Soc. Psychiatr. Psychiatr. Epidemiol. 33 (11), 568-578. 